\title{
MHD Numerical Simulation of Interdependent Flares and CMEs
}

\author{
Y.Z. Zhang ${ }^{1}$, J.X. Wang ${ }^{1}$, and Y.Q. $\mathrm{Hu}^{2}$ \\ ${ }^{1}$ National Astronomical Observatories, Chinese Academy of Sciences, Beijing 100012, China \\ email: zhangyingzhi@ourstar.bao.ac.cn \\ ${ }^{2}$ School of Earth and Space Sciences, University of Science and Technology of China, Hefei \\ 230026, China
}

\begin{abstract}
Using time-dependent resistive magnetohydrodynamic simulations, we study a flux rope eruption caused by magnetic reconnection with implication in coexistent flare-CME events. Without the resistance the flux rope system presents a double catastrophe. We use the first catastrophic state as the initial condition, in which an isolated flux rope coexists with two current sheets: a vertical one below and a transverse one above the flux rope. The flux rope erupts when reconnection starts in the current sheets, and the flux rope dynamics depends on the reconnection sequence in the two current sheets. We obtain three cases: reconnection occurs (1) simultaneously in the two current sheets, (2) first in the transverse one and then in the vertical, and (3) in an order opposite to case 2. Such a model exhibits characteristics of both magnetic breakout model for CME initiation and standard flare model. We argue that both breakout-like and tether-cutting reconnections may be important for CME eruptions.
\end{abstract}

Keywords. Sun: corona, Sun: coronal mass ejections (CMEs), Sun: flares

\section{Introduction}

Magnetic flux ropes are typical structures in the solar corona, and their eruptions are closely related to solar flares and coronal mass ejections (Low, 1993). Two types of models are popular in investigation of the solar eruptive phenomena. They are the standard flare model and magnetic breakout model. The standard model for the magnetic explosion in eruptive flares is first proposed by Sturrock (1966), and advanced by many latter studies. All of these studies represent that a cusp structure and two ribbon flares occur at low corona, and the reconnection is tether-cutting type at the internal current. Another type is breakout model (Antiochos et al., 1999) that involves multiple bipolar topology and requires external magnetic reconnection to occur on top of the sheared arcade. In their model the background field has a spherically symmetric quadrupolar field, rather than a dipolar field.

\section{Numerical Results}

In a recent study (Zhang, et al. 2005) we found that a double catastrophe exists for an isolated flux rope embedded in a quadrupolar background field. During the flux rope detaching from the solar surface the transverse current sheet above the flux rope expands quickly and the vertical current sheet below the flux rope grows elongated. Without reconnection the flux rope system may levitates in the corona.

However, at some time, the resistance occurs in the two current sheets and with difference reconnect sequence when not increasing the flux of the rope system. We have three cases, which are corresponding to three reconnection sequences, such as, the two 

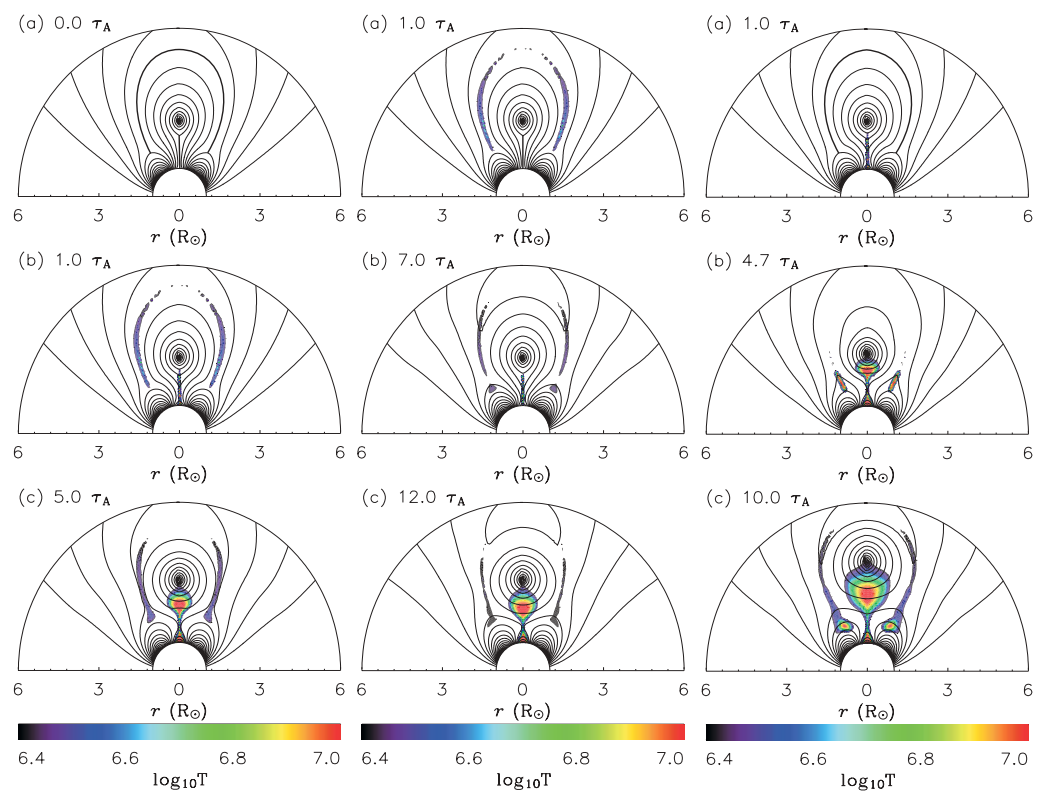

Figure 1. Case A (Left), Case B (Middle), Case C (Right). A cusp-shaped structure with high temperature is clearly seen after reconnection sets in across the vertical current sheet in three cases. Also, a high temperature structure appears in the corona right above the cusp structure.

current sheets have reconnected at the same time(case A), the transverse current sheet has reconnected firstly(case B), or the vertical current sheet has reconnected firstly(case C). We obtain the evolvement of the flux rope system in the corona in Figure 1.

\section{Conclusions}

The present model agrees with the breakout model (Antiochos, et al. 1999) if reconnection is started in the transversal current sheet, and the standard flare model (Chen and Shibata, 2000) if reconnection is started in the vertical current sheet. Nevertheless, we argue that both breakout-like external reconnections and tether-cutting internal reconnections are essential to the magnetic eruption in general. Our simulations just combine the two models together, which is probably more relevant to observations that many eruptive events occur in background fields of quadrupolar magnetic configuration (Sterling and Moore, 2004).

The work is supported by the National Natural Science Foundation of China (10233050, 40274049) and the National Key Basic Science Foundation (TG2000078404).

\section{References}

Antiochos, S. K., Devore, C. R., \& Klimchuk, J. A. 1999, ApJ 510, 485

Chen, P.F., \& Shibata, K. 2000, ApJ 545, 524

Low, B. C. 1993, ApJ 409,798

Sterling, A. C., \& Moore, R. L. 2004, ApJ 602, 1024

Sturrock, P.A. 1966, Nature 211, 695.

Zhang,Y.Z., Hu, Y.Q., \& Wang, J.X. 2005, ApJ 626,1096 